\title{
Approaching patients with irritable bowel syndrome
} Klaus Bielefeldt

Address: University of Pittsburgh Medical Center, Division of Gastroenterology, 200 Lothrop St, Pittsburgh, PA 15213, USA

Email: bielefeldtk@upmc.edu

Fl000 Medicine Reports 2010, 2:50 (doi:10.3410/M2-50)

The electronic version of this article is the complete one and can be found at: http://fl000.com/reports/medicine/content/2/50

\begin{abstract}
Treatment of functional gastrointestinal disorders remains difficult with many very different approaches showing similar response rates, regardless of whether they target luminal contents (e.g., presumed bacterial overgrowth), signaling within the gut wall (e.g., serotonin agonists or antagonists) or processing in the brain (e.g., cognitive behavioral therapy). Discrepancies between recent clinical trials and a meta-analysis have forced us to re-examine the use of antidepressants. Other studies have looked beyond the traditional drug therapies and have suggested other options such as dietary interventions and communication strategies that address relevant disease mechanisms and enable us to understand patient concerns, with the ultimate goal being to individualize and thus improve treatment outcomes.
\end{abstract}

\section{Introduction and context}

Symptoms of irritable bowel syndrome (IBS) are among the most common gastrointestinal complaints and lead to millions of consultative visits each year. While the third iteration of the Rome Consensus Conference provided yet another approach to positively diagnose functional illnesses, many physicians continue to see and approach IBS as a diagnosis of exclusion [1]. Once a diagnosis is made, we have to think about therapy, which may range from antibiotics for presumed small bowel bacterial overgrowth to serotonergic agonists or antagonists, depending on predominant symptoms and physician or patient preference. With spiraling healthcare costs and a highly prevalent syndrome, we need to ask ourselves whether we should standardize care and, in doing so, perhaps deliver more for less.

\section{Recent advances}

This call for standardized approaches and quality benchmarks led to development of guidelines that typically rely on evidence-based medicine and its primary tool, the meta-analysis. One such meta-analysis recently examined the utility of antidepressant medications in IBS. Many physicians use antidepressants when treating patients with functional bowel disorders. Mechanistic studies showing the influence of anxiety and/or hypervigilance on IBS symptoms may provide a rationale for this approach. Yet, prior studies have shown conflicting results, which could arguably be due to limited power and thus be clarified with a metaanalysis. A team of investigators thus compiled data from all published studies (identifying 13 studies that compared antidepressants with placebo) and concluded that antidepressants are effective in the treatment of IBS [2]. Although statistically convincing, a purely numerical approach computing an average across very different studies will gloss over any remaining conceptual concerns. One of the studies supporting the utility of these agents came from Iran, an area with male predominance in IBS, quite distinct from what physicians see in the US and European countries. The dosages of tricyclics vary five- to ten-fold, with the best effects seen in studies with the lowest dose. So, can we truly attribute such effects to the pharmacology of a medication and recommend a specific intervention (drug) if the effects cannot be explained by known mechanisms of action? This question becomes even more important when we look at negative results of recent trials examining the effects of citalopram in 
non-depressed adults with IBS or of amitriptyline in children with functional disorders $[3,4]$. Both trials were negative, not showing a difference between the active agent and placebo. Interestingly, pediatric patients showed impressive improvements in both arms (amitriptyline and placebo), demonstrating the power of placebo. Recently, a study did suggest that imipramine may be effective in the treatment of IBS [5], however, another demonstrated no significant benefit for paroxetine [6], further highlighting the differences in the results within this field. So, why do we see discrepancies between these studies and the metaanalysis? Perhaps if rigor went beyond methodological approaches and transparency to include conceptual assessments, less disparity would be seen. IBS is defined by a wide range of symptoms with likely very different pathophysiologies. It may be a clinically useful label, but the term describes a syndrome, not disease mechanisms. Rather than relying on algorithmic approaches that presume similar disease mechanisms and applying similar treatments to every person, no matter whether diarrhea, constipation, bloating or pain predominate, we need to practice 'personalized' medicine. In this context, one may well use antidepressants, basing decisions on the relative contribution of affect and choosing an agent that fits best in terms of effect and side-effect profile.

The study by Saps and colleagues [4] highlighted the placebo effect, which in most large IBS trials is lower than in this study, but still ranges around $40 \%$. The importance of placebo responses has triggered investigations into the different components of this powerful effect. An interesting study focused on us, the healthcare providers. The training we go through largely focuses on the content of our discussions: what is going on, what treatments we recommend and why we suggest them, rather than how we relay that information to the patient. As part of a trial on acupuncture in IBS, a team of investigators paired sham treatment with different communicative strategies [7]. One group met a qualified therapist who explained the treatment plan, the other talked to a person who employed empathy, active listening and instilled confidence. Active listening, empathy and confidence 'augmented' the placebo effect of acupuncture alone, and therefore may matter more than the technical competence that we can claim as certified specialists [7]. The results remind us that therapy starts even before the first pill has been taken. Our interactions with IBS patients strongly influence the effect of our interventions. Such findings may well confirm what we already thought, yet, even if the results may not surprise us, we as healthcare providers should more effectively employ these communicative skills, as patients seemingly continue to leave our offices not feeling listened to, not understanding their illness or not feeling understood [8,9]. In a small study, Collins et al. [8] examined whether expectations were met during consultative visits with gastroenterologists. Despite a focus on physicians with special interest and expertise in functional bowel diseases, many patients remained unsure or unconvinced about their diagnosis. Halpert and colleagues [9] chose a different approach and surveyed IBS patients using the internet. Most participants looked for education, explanation, empathy and engaged, active listening from physicians, mirroring the very components that contribute to the placebo response. Unfortunately, they also saw most healthcare providers as lacking in these very domains. Perhaps these studies give us a benchmark for quality improvements that are more applicable than those one can obtain from metaanalyses.

If the patients or physicians do not want to use pharmacotherapy, there are other options available, ranging from herbal remedies to dietary changes and psychologically-based interventions. When considering food intake, high-fiber diets seem to be indelibly linked to improvements in IBS, especially IBS coexisting with constipation, with many reviews and books recommending it as one of the pillars of IBS management. But does it truly improve patients' quality of life, which should be our primary goal in persons with functional gastrointestinal disorders? A recent study demonstrated that insoluble fiber (bran) was not better than placebo, and although soluble fiber (psyllium) transiently helped, this benefit was lost at the end of the 3-month study [10]. The minor and brief statistical difference does not translate into clinical relevance, especially considering the chronic nature of IBS. The results should prompt us to look for correlations between a patient's symptoms and their intake and output (or outcome) before automatically recommending changes in fiber intake. Fiber intake is only one of the many complex components that make up our diet, so we should not stop obtaining information about other food and drink intake, or promoting healthy nutrition, and should perhaps even think about fiber as a 'prebiotic.' Yet, this study should lead us to question the seemingly automatic assumption that increasing fiber intake will improve IBS.

Besides fiber, intake of other foods can also affect IBS symptoms, with many patients reporting intolerances 
or 'trigger foods.' A group of Italian investigators tried to find an objective correlate for such subjective intolerances and examined the role of food allergies in IBS [11]. Using elimination diets and food challenges, about one-third of their patients reacted to cow milk or wheat protein. Roughly half of these patients may have suffered from a true food allergy, which was operationally defined by activation of basophils in vitro. Thus, food allergies may not be very common in IBS patients, but this study at least suggests that they may be more common than other illnesses often associated with IBS, such as celiac disease. The basophil activation assay is not widely available and confirmatory studies are certainly needed. However, if proven to be effective, we may need to make use of strategies such as this assay because patient history, effects of elimination diets and food challenge, as well as serum IgE levels against suspected antigens, poorly predict the potential allergic basis of food intolerance. If carbohydrates from fructose and gluten-containing substances do indeed significantly contribute to IBS symptoms, as this and some other studies of elimination diets suggest, one could take a different approach and eliminate carbohydrates completely. In a small, uncontrolled study, Austin and colleagues [12] asked patients with refractory functional diarrhea to follow a strict carbohydrate exclusion diet. The rigid approach was difficult, required implementation through a clinical research center, prompted several drop outs and may not be desirable as it is, by definition, nutritionally imbalanced. However, it also led to improvement in more than two-thirds of those who complied. While the popularity of the Atkins and South Beach diets may have dropped in recent years, less stringent strategies may not be quite as effective but could be more easily implemented, still resonate with many patients and may well provide secondary benefits in diarrheapredominant IBS patients by targeting the spreading obesity epidemic [12].

\section{Implications for clinical practice}

In 2010, we may need to split more and look to original data, rather than lumping and considering only the meta-analyses. Paying attention to the patient's primary symptoms and their main concerns should be made. The contribution of dietary or other lifestyle choices to their symptoms should be considered, as should the role of affect. While trying to obtain this information, physicians need to focus on more than just the content of their discussion and hone in on communicative strategies that may not only increase patients satisfaction, but also improve outcome. Clinical trials have to use rigor and systematic strategies to test hypotheses and understand and/or treat possible mechanisms of disease. In poorly understood complex syndromic illnesses with divergent clinical manifestations and likely divergent underlying mechanisms such as IBS, the clinician may need to abandon evidence-based medicine and rely instead on individualized approaches aimed at increasing quality of life.

\section{Abbreviation}

IBS, irritable bowel syndrome.

\section{Competing interests}

The author declares that he has no competing interests.

\section{References}

I. Spiegel BMR, Farid M, Esrailian E, Talley J, Chang L: Is irritable bowel syndrome a diagnosis of exclusion? A survey of primary care providers, gastroenterologists, and IBS experts. Am J Gastroenterol 2010, I05:848-58.

2. Ford AC, Talley NJ, Schoenfeld PS, Quigley EMM, Moayyedi P: Efficacy of antidepressants and psychological therapies in irritable bowel syndrome: systematic review and metaanalysis. Gut 2009, 58:367-78.

3. Ladabaum U, Sharabidze A, Levin TR, Zhao WK, Chung E, Bacchetti P, Jin C, Grimes B, Pepin C]: Citalopram is not effective therapy for non-depressed patients with irritable bowel syndrome. Clin Gastroenterol Hepatol 2010, 8:42-8.

FI000 Factor 3.0 Recommended Evaluated by Klaus Bielefeldt 09 Oct 2009

4. Saps M, Youssef N, Miranda A, Nurko S, Hyman P, Cocjin J, Di Lorenzo C: Multicenter, randomized, placebo-controlled trial of amitriptyline in children with functional gastrointestinal disorders, gastroenterology. Gastroenterology 2009, |37:|26|-9.

FI000 Factor 6.4 Must Read

Evaluated by Klaus Bielefeldt 27 Jul 2009, Jon Markowitz 26 Oct 2009

5. Abdul-Baki H, El Hajj II, Elzahabi L, Azar C, Aoun E, Skoury A, Chaar $\mathrm{H}$, Sharara $\mathrm{Al}$ : A randomized controlled trial of imipramine in patients with irritable bowel syndrome. World J Gastroenterol 2009, I 5:3636-42.

6. Masand PS, Pae CU, Krulewicz S, Peindl K, Mannelli P, Varia IM, Patkar AA: A double-blind, randomized, placebo-controlled trial of paroxetine controlled-release in irritable bowel syndrome. Psychosomatics 2009, 50:78-86.

7. Kaptchuk TJ, Kelley JM, Conboy LA, Davis RB, Kerr CE, Jacobson EE, Kirsch I, Schyner RN, Nam BH, Nguyen LT, Park M, Rivers AL, McManus C, Kokkotou E, Drossman DA, Goldman P, Lembo AJ: Components of placebo effect: randomised controlled trial in patients with irritable bowel syndrome. BMJ 2008, 336:9991003.

FI000 Factor 6.8 Must Read

Evaluated by Chris Del Mar 17 Apr 2008, Klaus Bielefeldt 24 Apr 2008, Roberto De Giorgio 0I May 2008, Mellar Davis 30 Jun 2008, Mike Cummings I5 Jul 2008

8. Collins J, Farrall E, Turnbull DA, Hetzel DJ, Holtmann G, Andrews JM: Do we know what patients want? The doctor-patient communication gap in functional gastrointestinal disorders. Clin Gastroenterol Hepatol 2009, 7:I252-I254.e2.

FI000 Factor 3.0 Recommended

Evaluated by Klaus Bielefeldt 2 I Jul 2009 
9. Halpert A, Dalton CB, Palsson O, Morris C, Hu Y, Bangdiwala S, Hankins J, Norton N, Drossman DA: Irritable bowel syndrome patients' ideal expectations and recent experiences with healthcare providers: a national survey. Dig Dis Sci 20I0, 55: 375-83.

10. Bijkerk CJ, de Wit NJ, Muris JWM, Whorwell PJ, Knottnerus JA, Hoes AW: Soluble or insoluble fibre in irritable bowel syndrome in primary care? Randomised placebo controlled trial. BMJ 2009, 339:b3I54.

Changes Clinical Practice

FI000 Factor 6.0 Must Read

Evaluated by Anton Emmanuel 12 Oct 2009
II. Carroccio A, Brusca I, Mansueto P, Pirrone G, Barrale M, Di Prima L Ambrosiano G, lacono G, Lospalluti ML, La Chiusa SM, Di Fede G: A cytologic assay for diagnosis of food hypersensitivity in patients with irritable bowel syndrome. Clin Gastroenterol Hepatol 2010, 8:254-60.

12. Austin GL, Dalton CB, Hu Y, Morris CB, Hankins J, Weinland SR, Westman EC, Yancy Jr WS, Drossman DA: A very lowcarbohydrate diet improves symptoms and quality of life in diarrhea-predominant irritable bowel syndrome. Clin Gastroenterol Hepatol 2009, 7:706-708.el.

FI000 Factor 3.0 Recommended

Evaluated by Klaus Bielefeldt 18 Mar 2009 